\title{
Florfenicol concentrations in ovine tear fluid following intramuscular and subcutaneous administration and comparison with the minimum inhibitory concentrations against mycoplasmal strains potentially involved in infectious keratoconjunctivitis
}

\author{
Alain Regnier, Dr Med Vet, PhD; Valérie Laroute, PhD; Anne Gautier-Bouchardon, PhD; \\ Véronique Gayrard, Dr Med Vet, PhD; Nicole Picard-Hagen, Dr Med Vet, PhD; \\ Pierre-Louis Toutain, Dr Med Vet, PhD
}

\begin{abstract}
Objective - To measure florfenicol concentrations in ovine tear fluid after IM and SC administration and determine minimum inhibitory concentrations (MICs) of florfenicol against field isolates of Mycoplasma organisms potentially involved in infectious keratoconjunctivitis.

Animals -9 healthy adult Lacaune ewes.

Procedures_-Animals received an IM and SC administration of florfenicol $(20 \mathrm{mg} / \mathrm{kg})$ in a 2 -way crossover design. Samples of blood and tear fluid were collected before and for 24 hours after administration. Concentrations of florfenicol in plasma and tear fluid were measured via high-performance liquid chromatography. The MIC of florfenicol for various Mycoplasma strains cultured from sheep and goats was determined via an agar dilution method.

Results-Mean florfenicol concentration in tear fluid for the 24-hour period was significantly higher after IM administration $(0.70 \mu \mathrm{g} / \mathrm{mL})$ than after SC administration $(0.22 \mu \mathrm{g} / \mathrm{mL})$ and was maintained for a longer duration. The lacrimal fluid-to-plasma concentration ratio was not different between the 2 routes of administration, with mean values of $40.2 \%$ and $32.5 \%$ after IM and SC administration, respectively. The MIC for Mycoplasma agalactiae, Mycoplasma conjunctivae, and Mycoplasma mycoides isolates ranged from 0.5 to $8 \mu \mathrm{g}$ of florfenicol/mL. Two strains of $M$ agalactiae could be considered resistant to florfenicol.

Conclusions and Clinical Relevance-Florfenicol readily penetrated the preocular tear fluid of sheep after IM and SC administration. For both routes of administration, doses > 20 $\mathrm{mg} / \mathrm{kg}$ would be necessary to achieve tear fluid concentrations of florfenicol greater than the MICs for most strains of Mycoplasma organisms. (Am J Vet Res 2013;74:268-274)
\end{abstract}

$\mathbf{I}^{\mathrm{n}}$

nfectious keratoconjunctivitis (contagious ophthalmia) is a contagious ocular disease that affects sheep, ${ }^{1-3}$ goats, and wild small ruminants. ${ }^{4,5}$ The disease manifests with acute clinical signs of conjunctivitis and, in its later stages, keratitis with corneal edema, vascularization, and possibly ulceration. ${ }^{6,7}$ Spread through a flock is rapid, and the most severely affected eyes can progress to extensive corneal abscesses and panoph-

\footnotetext{
Received January 31, 2012.

Accepted June 7, 2012.

From UMR 181 Physiopathologie et Toxicologie Expérimentales, INRA, Ecole Nationale Vétérinaire, 23 chemin des Capelles, B.P. 87614, 31076 Toulouse Cedex 03, France (Regnier, Gayrard, Picard-Hagen, Toutain); INRA, UMR 792 Ingénierie des Systèmes Biologiques et des Procédés, LISB-INSA, 135 avenue de Rangueil, 31077 Toulouse Cedex 04, France (Laroute); and ANSES, Unité de Mycoplasmologie-Bactériologie, BP 53, 22440 Ploufragan, France (Gautier-Bouchardon).

Presented in abstract form at the 39th Annual Meeting of the American College of Veterinary Ophthalmologists, Boston, October 2008. Address correspondence to Dr. Regnier (a.regnier@envt.fr).
}

\begin{tabular}{|c|c|}
\hline & AbBreViations \\
\hline$A \cup C_{\text {plasma }}$ & $\begin{array}{l}\text { Area under the drug concentration-time } \\
\text { curve in plasma }\end{array}$ \\
\hline$A U C_{\text {tears }}$ & $\begin{array}{l}\text { Area under the drug concentration-time } \\
\text { curve in tear fluid }\end{array}$ \\
\hline Cmax & Maximum concentration \\
\hline $\mathrm{MIC}$ & Minimum inhibitory concentration \\
\hline Tmax & Time of maximum concentration \\
\hline
\end{tabular}

thalmitis. ${ }^{8}$ Although infectious keratoconjunctivitis in sheep is ascribed to various agents such as Branhamella ovis and Chlamydia spp, ${ }^{6,9}$ data in the literature suggest that Mycoplasma spp play a primary causal role in the disease ${ }^{10-12}$ Mycoplasma conjunctivae is a common isolate in outbreaks of the disease, and ocular instillations of pure cultures of this organism can reproduce the disease. ${ }^{2,11-15}$ Other Mycoplasma spp such as Mycoplasma agalactiae and Mycoplasma mycoides subsp mycoides have been isolated from sheep with keratocon- 
junctivitis and other manifestations of the disease..$^{7,16}$ Topically or parenterally administered antimicrobials, such as tetracycline or oxytetracycline, are used in severely affected animals to shorten the clinical course of the disease and reduce the risk of permanent corneal opacification. ${ }^{6,7,9}$

The rationale for systemic administration of antimicrobials in the treatment of infectious keratoconjunctivitis in sheep has been based on the observation that some antimicrobial agents can diffuse into the lacrimal fluid after parenteral administration ${ }^{17}$ and that this route of administration can be clinically effective. ${ }^{9,18}$ Florfenicol, a monofluorinated analogue to thiamphenicol, has greater in vitro activity against pathogenic bacteria than does chloramphenicol or thiamphenicol. ${ }^{19,20}$ In sheep, the drug has a high bioavailability after IM administration, ${ }^{21,22}$ a prolonged half-life of elimination after IM and SC administration, ${ }^{22,23}$ and a large volume of distribution $(1.86 \mathrm{~L} / \mathrm{kg})$, which suggests good diffusion into body tissues. ${ }^{22}$ This last feature partly results from the low amount of binding to plasma proteins at therapeutic concentrations, ${ }^{24,25}$ which is an important determinant for diffusion into tears. ${ }^{26,27}$ Excretion of parenterally administered florfenicol in the tear fluid is not confirmed, but studies ${ }^{28-30}$ have found that IM administration of florfenicol is effective in the treatment of infectious keratoconjunctivitis in cattle. Given the possible use of florfenicol in the treatment of infectious keratoconjunctivitis in sheep, the objective of the study reported here was to evaluate the drug's penetration in ovine tears after a single IM and SC administration and determine its MIC for various Mycoplasma spp potentially involved in this disease.

\section{Materials and Methods}

Animals-Nine healthy Lacaune sheep that weighed 45 to $57 \mathrm{~kg}$ and were 2 to 3 years old were included in the study. All animals were nonpregnant females that were allowed to acclimate to their surroundings for at least 2 weeks prior to the start of the study. During the acclimation and study periods, the ewes were housed separately in metabolism cages. They were provided hay and water ad libitum and fed a commercially prepared concentrate mixture once daily. A general physical examination and an ophthalmic examination were performed on each ewe during the acclimation period to rule out preexisting systemic and ocular abnormalities. All animal experiments were performed in compliance with institutional and national guidelines in accordance with the European Community Council directive 86/609/EEC. The experimental protocol was approved by the INRA Toulouse/ENVT ethics committee.

Experimental design-The study was conducted in a crossover design, in which each sheep received florfenicol by the 2 routes of administration in a random order and with a washout period of at least 1 week between the 2 administrations. Florfenicol ${ }^{\mathrm{a}}$ was administered at a dose of $20 \mathrm{mg} / \mathrm{kg}$ for each route of administration; all treatments were administered between 9:00 AM and 9:30 AM. The drug was administered IM and SC in the right gluteal muscle mass and in the right lateral abdominal region (flank), respectively, with a 19-gauge needle.

For each route of administration, blood and tear fluid samples were collected from each sheep before (time 0 ) and $0.25,0.50,1,2,3,4,6,8,10$, and 24 hours after florfenicol administration. Blood samples (3 mL) were collected via a 17-gauge polyethylene catheter implanted in the left jugular vein 1 day before drug administration and sample collection. The catheter was flushed with saline $(0.9 \% \mathrm{NaCl})$ solution containing heparin $(10 \mu \mathrm{g} / \mathrm{mL})$ after each sample. Blood samples were transferred into heparinized tubes, and the plasma was separated by centrifugation $(1,000 \times g$ for 10 minutes at $\left.4^{\circ} \mathrm{C}\right)$ and stored frozen $\left(-20^{\circ} \mathrm{C}\right)$ in polypropylene tubes until analysis. Tear fluid specimens were collected without the use of topical anesthesia, as described elsewhere, ${ }^{31,32}$ with preweighed Schirmer tear strips placed in the tear fluid between the middle and inner third of the lower lid of 1 randomly selected eye of each sheep. Collection was limited to 30 seconds but was stopped before then if the strip became saturated with at least $20 \mathrm{~mm}$ of fluid. Each strip was then returned to its corresponding vial and was immediately reweighed. Tear samples were stored frozen at $-20^{\circ} \mathrm{C}$ until analysis.

Analytic procedures-Samples of plasma and tear fluid were analyzed for florfenicol concentrations via high-performance liquid chromatography. An internal standard (prednisolone $[0.2 \mu \mathrm{g} / \mathrm{mL}])^{\mathrm{b}}$ and florfenicol were extracted from plasma samples via solid-phase extraction. The high-performance liquid chromatography apparatus consisted of a pump system equipped with an automatic injector and a UV detector set at a wavelength of $224 \mathrm{~nm}$. Separation was achieved via a reversephase column ${ }^{\mathrm{c}}$ with a guard column. ${ }^{\mathrm{d}}$ The mobile phase consisted of a mixture of distilled water and acetonitrile (74 parts distilled water:26 parts acetonitrile) and was used at a flow rate of $0.7 \mathrm{~mL} / \mathrm{min}$. For these conditions, florfenicol and prednisolone were eluted at retention times of 7.9 and 10.3 minutes, respectively. The quantification limit of the method was $0.2 \mu \mathrm{g} / \mathrm{mL}$. Accuracy of the assay ranged from $96 \%$ to $102 \%$, and coefficients of variation for interday and intraday precision were $<5 \%$ for both plasma and tear fluid.

For determination of florfenicol concentrations in tear fluid, $1 \mathrm{~mL}$ of internal standard (prednisolone $[0.2 \mu \mathrm{g} / \mathrm{mL}])$ in acetonitrile was added to vials containing the preweighed Schirmer strips impregnated with standard solutions of florfenicol or tear fluids. After evaporation, the dry extract was redissolved in $40 \mu \mathrm{L}$ of acetonitrile, and $20 \mu \mathrm{L}$ of the solution then was injected. For the tear fluid, accuracy was 97\% to 104\% for intraday variation and $<10 \%$ for interday variation; the limit of quantification was $0.25 \mu \mathrm{g} / \mathrm{mL}$. Results obtained for the tear fluid were expressed as micrograms per milliliter, assuming a tear specific gravity of 1 , as reported elsewhere. ${ }^{33}$

Pharmacokinetic analysis-Pharmacokinetic parameters, except for terminal half-life, were determined via noncompartmental analysis. Terminal halflife after IM administration was estimated via linear regression. The Cmax and Tmax were obtained from 
the raw data. The AUC and mean residence time were calculated via the arithmetic trapezoidal rule for the experimental period (0 to 24 hours). Drug concentrations in tear fluid were analyzed via a noncompartmental approach. Calculated values included the AUC $C_{\text {tears }}$ estimated from time 0 to the last measurable concentration in tear fluid, Cmax in tear fluid, and Tmax in tear fluid. The extent of penetration of florfenicol into the tear fluid was calculated by dividing $\mathrm{AUC}_{\text {tears }}$ by the corresponding $\mathrm{AUC}_{\text {plasma }}$ and was reported as a percentage.

MIC of florfenicol for Mycoplasma isolates from small ruminants-Five strains of $M$ mycoides subsp mycoides large colonies, 5 strains of $M$ conjunctivae, and 22 strains of $M$ agalactiae isolated from sheep or goats were used in the study. One reference strain of each species was also evaluated.

Strains were grown in Friis broth medium containing a dye indicator (ie, phenol red), which allowed detection of the metabolic activity of Mycoplasma organisms and therefore their growth. ${ }^{34}$ Strains were incubated at $37^{\circ} \mathrm{C}$ until there was a relevant color change (from pink to orange-yellow). After addition of $20 \%$ (vol/vol) sterile glycerol, ${ }^{\mathrm{e}}$ the cultures were separated into aliquots and stored at $-70^{\circ} \mathrm{C}$. These cultures were subsequently titrated on Friis agar medium to determine the number of CFUs per milliliter.

The antimicrobial solutions for MIC determinations were prepared by dissolving florfenicol ${ }^{\mathrm{f}}$ in absolute alcohol and subsequently diluting that solution in
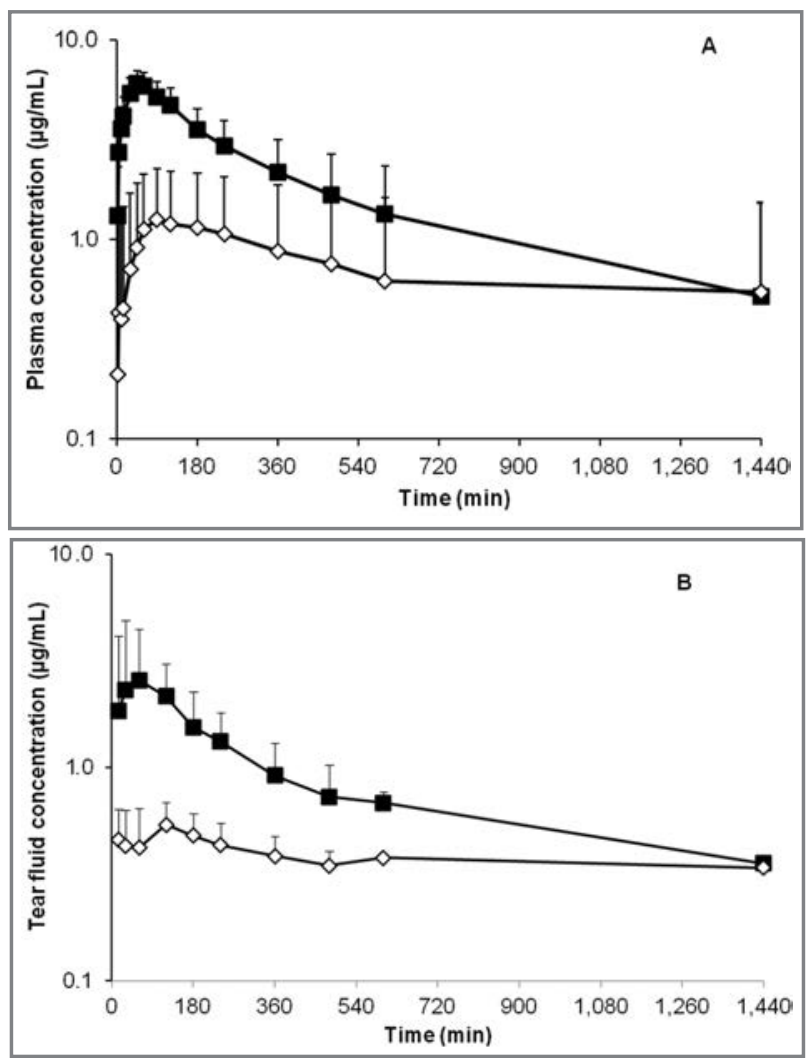

Figure 1-Mean \pm SD florfenicol concentrations in plasma (A) and tear fluid (B) obtained from 9 sheep before (time 0 ) and at various times after IM (black squares) and SC (white diamonds) administration of $20 \mathrm{mg}$ of florfenicol/kg. water. Solutions were sterilized by filtration through filters with a pore size of $0.22 \mu \mathrm{m} .^{\mathrm{g}}$

The MICs were determined by an agar dilution method on Friis medium. ${ }^{35,36}$ Florfenicol concentrations ranged between 0.03 and $64 \mu \mathrm{g} / \mathrm{mL}$. A multipoint inoculator $^{\mathrm{h}}$ was used to deposit $1 \mu \mathrm{L}$ of mycoplasmal suspension ( $10^{5} \mathrm{CFUs} / \mathrm{mL}$ ) on each florfenicol concentration. In addition, the strains were incubated on an agar medium that did not contain antimicrobials. Plate contents were incubated at $37^{\circ} \mathrm{C}$ in a $5 \% \mathrm{CO}_{2}$ atmosphere for 3 to 5 days (depending on the rapidity of growth of the strains tested). The MIC was defined as the lowest concentration for which no visible growth was observed. Strains were considered susceptible to florfenicol when the MIC was $\leq 4 \mu \mathrm{g} / \mathrm{mL}$.

Statistical analysis-Data were reported as mean \pm SD. Differences between the pharmacokinetic variables for the IM and SC routes of administration were compared via paired $t$ tests. For all comparisons, values of $P$ $<0.05$ were considered significant.

\section{Results}

No adverse effects were observed after florfenicol administration, and all sheep remained healthy throughout the study. Plasma florfenicol kinetic profiles after IM and SC administrations were plotted (Figure 1). The profiles revealed that the absorption rate of florfenicol was significantly faster and plasma drug concentrations were significantly higher for the IM route than for the SC route. A mean \pm SD Cmax of $6.23 \pm$ $6.16 \mu \mathrm{g} / \mathrm{mL}$ was detected in plasma at $55 \pm 26 \mathrm{~min}-$ utes after IM administration, compared with a mean Cmax of $1.32 \pm 0.54 \mu \mathrm{g} / \mathrm{mL}$ at $130 \pm 58$ minutes after SC administration. The mean terminal half-life was 544 \pm 104 minutes after IM administration and was longer after SC administration, but it could not be calculated for SC administration because the terminal portion of the curve was flat. Other major pharmacokinetic determinants derived from data collected after IM and SC administrations were summarized (Table 1).

Tear fluid concentration-time profiles for florfenicol after IM and SC administrations were plotted (Figure 1). Pharmacokinetic parameters in tear fluid determined after IM and SC administration of florfenicol were summarized (Table 2). Overall, florfenicol concentrations in tear fluid were higher and persisted longer after IM administration than after SC administration. A mean \pm SD Cmax of $2.74 \pm 2.72 \mu \mathrm{g} / \mathrm{mL}$ was detected in tear fluid after IM administration, which

Table $1-$ Mean \pm SD values describing the disposition of florfenicol in plasma obtained from 9 sheep after a single IM or SC administration at a dose of $20 \mathrm{mg} / \mathrm{kg}$.

\begin{tabular}{|lcc|}
\hline Variable & IM administration & SC administration \\
\hline Cmax $(\mu \mathrm{g} / \mathrm{mL})$ & $6.23 \pm 6.16$ & $1.32 \pm 0.54^{*}$ \\
Tmax $(\mathrm{min})$ & $55 \pm 26$ & $130 \pm 58^{*}$ \\
AUCplasma $(\mu \mathrm{g} \bullet \mathrm{min} / \mathrm{mL})$ & $2,544 \pm 1,041$ & $990 \pm 434^{*}$ \\
$\mathrm{MRT}_{\text {0-last }}(\mathrm{min})$ & $438 \pm 74$ & $575 \pm 121^{*}$ \\
\hline
\end{tabular}

*Value differs significantly $(P=0.01)$ from the value for IM administration.

$\mathrm{MRT}_{\text {0-last }}=$ Mean residence time from the time of florfenicol administration (time 0 ) to the time of the last measurable concentration. 
Table 2-Mean \pm SD values describing the disposition of florfenicol in tear fluid obtained from 9 sheep after a single IM or SC administration at a dose of $20 \mathrm{mg} / \mathrm{kg}$.

\begin{tabular}{lcc}
\hline Variable & IM administration & SC administration \\
\hline $\mathrm{Cmax}(\mu \mathrm{g} / \mathrm{mL})$ & $2.74 \pm 2.72$ & $0.56 \pm 0.19^{*}$ \\
$\mathrm{Tmax}_{\mathrm{m}}(\mathrm{min})$ & $90 \pm 37$ & $127 \pm 56$ \\
$\mathrm{AUC}_{\text {tears }}(\mu \mathrm{g} \bullet \mathrm{min} / \mathrm{mL})$ & $1,009 \pm 355$ & $314 \pm 243^{*}$ \\
$\mathrm{~T}_{\text {last }}(\mathrm{min})$ & 1,053 & 787 \\
$\mathrm{C}_{\text {last }}(\mu \mathrm{g} / \mathrm{mL})$ & 0.45 & 0.34 \\
Mean $\mathrm{concentration}_{\text {in tear fluid }}(\mu \mathrm{g} / \mathrm{mL})$ & $0.70 \pm 0.24$ & $0.22 \pm 0.17^{*}$ \\
$\mathrm{AUC}_{\text {tears }}: \mathrm{AUC}_{\text {plasma }}$ ratio & $0.40 \pm 0.08$ & $0.32 \pm 0.26$ \\
\hline
\end{tabular}

*Value differs significantly $(P<0.05)$ from the value for IM administration.

$\mathrm{C}_{\text {last }}=$ Last measurable drug concentration in tear fluid. $\mathrm{T}_{\text {last }}=$ Time of last measurable drug concentration in tear fluid.

Table 3-The MICs $(\mu \mathrm{g} / \mathrm{mL})$ for florfenicol against field and type strains of 3 Mycoplasma spp isolated from small ruminants in France.

\begin{tabular}{|lccccc}
\hline & & \multicolumn{3}{c}{ Field strains } \\
\cline { 3 - 6 } Mycoplasma spp & $\begin{array}{c}\text { Type } \\
\text { strains* }\end{array}$ & Range & MIC $_{50}$ & MIC $_{90}$ \\
\hline Mycoplasma mycoides subsp & 4 & 4 & 4 & 4 \\
mycoides large colonies $(\mathrm{n}=6)$ & & & & \\
Mycoplasma conjunctivae $(\mathrm{n}=6)$ & 2 & $2-4$ & 2 & 4 \\
Mycoplasma agalactiae $(\mathrm{n}=23)$ & 0.5 & $1-8$ & 1 & 4
\end{tabular}

*Type strains were $Y$ Goat for $M$ mycoides subsp mycoides large colonies, National Collection of Type Cultures 10123 for $M$ conjunctivae, and ATCC 25834 for $M$ agalactiae.

$\mathrm{MIC}_{50}=$ The MIC required to inhibit growth of $50 \%$ of strains. $\mathrm{MIC}_{90}=$ The MIC required to inhibit growth of $90 \%$ of strains.

was significantly higher than that of $0.56 \pm 0.19 \mu \mathrm{g} /$ $\mathrm{mL}$ detected after SC administration. By contrast, the Tmax in tear fluid was not significantly different between the 2 routes of administration, with mean values of $90 \pm 37$ minutes and $127 \pm 56$ minutes after IM and $\mathrm{SC}$ administration, respectively. A mean $\mathrm{AUC}_{\text {tears }}$ of $1,009 \pm 355 \mu \mathrm{g} \bullet \mathrm{min} / \mathrm{mL}$ was calculated for IM administration, which was significantly higher, compared with that of $314 \pm 243 \mu \mathrm{g} \bullet \mathrm{min} / \mathrm{mL}$ calculated for SC administration. The mean drug concentration in tears over the 24-hour period was significantly higher after IM administration $(0.70 \pm 0.24 \mu \mathrm{g} / \mathrm{mL})$ than after SC administration $(0.22 \pm 0.17 \mu \mathrm{g} / \mathrm{mL})$. The mean time of the last measurable drug concentration in tear fluid was 1,053 minutes (range, 480 to 1,440 minutes) and 787 minutes (range, 240 to 1,440 minutes) after IM and SC administration, respectively. No significant difference was found between the mean florfenicol tear penetration calculated for IM (40.2\%) and SC (32.5\%) administration.

All the $M$ conjunctivae and $M$ mycoides strains were susceptible to florfenicol, with MICs between 2 and $4 \mu \mathrm{g} / \mathrm{mL}$ (Table 3). Results obtained for $M$ agalactiae were less homogenous, with MICs ranging from $0.5 \mu \mathrm{g} /$ $\mathrm{mL}$ for the type strains to $8 \mu \mathrm{g} / \mathrm{mL}$ for 2 recently isolated field strains.

\section{Discussion}

Antimicrobials that can be used to treat infectious keratoconjunctivitis in sheep must have a broad spectrum of activity to cover the wide variety of causative agents, in- cluding Mycoplasma spp, and must provide effective concentrations in the tear fluid after systemic administration. The study reported here was designed to assess the secretion of florfenicol into tear fluid after parenteral administration in sheep and correlate these drug concentrations with the MICs against Mycoplasma spp to make predictions on the therapeutic value of systemic administration of florfenicol for the treatment of infectious keratoconjunctivitis in sheep by use of this pharmacokinetic-pharmacodynamic relationship. Although florfenicol is not labeled for use in sheep, pharmacokinetics after IM and SC administration in this species with doses ranging from 20 to $40 \mathrm{mg} /$ $\mathrm{kg}$ have been reported. ${ }^{21-23}$ The differences in the blood kinetic behavior of florfenicol between the IM and SC routes of administration in the present study are in agreement with results of those studies. ${ }^{21-23}$ The disposition of florfenicol after IM administration was characterized by a more rapid absorption, a higher plasma $C \max$, and a higher relative bioavailability, compared with the disposition after SC administration. The mean terminal half-life and mean residence time after IM administration were in agreement with findings in other studies, ${ }^{21,22}$ which suggested that IM injection should be the selected mode for florfenicol administration in sheep.

The data in the present study also illustrated the ability of parenterally administered florfenicol to be secreted into the tear fluid of sheep. The mean penetration of florfenicol into ovine tear fluid was $40.2 \%$ and $32.5 \%$ after IM and SC administration, respectively. By comparison, the amount of tear secretion of kanamycin after IM administration in calves was $9 \%,{ }^{37}$ and mean tear plasma ratios of $6.2 \%, 9.4 \%, 8.4 \%$, and $4.3 \%$ have been reported in humans after IV administration of gentamicin, tobramycin, amikacin, and netilmicin, respectively. ${ }^{38}$ Similarly, parenteral administration of a long-acting formulation of oxytetracycline led to low drug concentrations in tear fluid of sheep and calves..$^{17,39}$ Oxytetracycline concentrations in lacrimal fluid were one-seventh to one-tenth those in serum after IV administration in sheep ${ }^{17}$ and were $<1 \mu \mathrm{g} / \mathrm{mL}$ even at serum Cmax following IM administration in calves. ${ }^{39}$

The ability of florfenicol to distribute into tears may be related to its large volume of distribution, lipophilicity, and low protein binding, given that these features are indicators of diffusion of systemically administered drugs in the eyes and CNS. ${ }^{27,38}$ A study ${ }^{40}$ of CSF obtained from calves after IV administration of florfenicol $(20 \mathrm{mg} / \mathrm{kg})$ revealed that the ratio between the area under the curve in CSF and plasma was $46 \%$, a value close to the value of $40.2 \%$ calculated in the present study for the $\mathrm{AUC}_{\text {tears }}: \mathrm{AUC}_{\text {plasma }}$ ratio after IM administration.

The Schirmer strip technique has been used for tear collection to study the pharmacokinetic profile of ofloxacin, ciprofloxacin, norfloxacin, and tobramycin in human tear fluid ${ }^{31,32}$ and was found to have a comparable accuracy and precision to collection of tears with capillary glass tubes or surgical sponges for quantifying drug concentrations in tear fluid. ${ }^{33}$ The Schirmer strip technique was used in the present study because it is easy to perform, can be performed rapidly, and does not cause adverse ocular effects when used repeatedly to obtain samples for pharmacokinetic evaluations. ${ }^{27}$ 
Strains isolated from sheep and goats were used for MIC determination because closely related strains circulate between these 2 species in field conditions. ${ }^{41,42}$ The MIC results were similar between sheep and goat strains (data not shown). Currently, there are no MIC breakpoint values approved by the Clinical Laboratory Standard Institute for Mycoplasma infections of domestic animals and humans, ${ }^{43}$ and few studies ${ }^{44-47}$ have been conducted on antimicrobial susceptibility of $M$ agalactiae and $M$ mycoides subsp mycoides large colonies. Strains of these 2 Mycoplasma spp were susceptible to various antimicrobials in vitro, ${ }^{45-47}$ but isolates of M agalactiae with elevated MICs have also been identified. ${ }^{47}$

To our knowledge, antimicrobial susceptibilities of $M$ conjunctivae strains have not been investigated. In the study reported here, the MICs of florfenicol for $M$ agalactiae, $M$ conjunctivae, and $M$ mycoides subsp mycoides isolates ranged from 0.5 to $8 \mu \mathrm{g} / \mathrm{mL}$. Florfenicol MICs obtained in the present study for $M$ mycoides subsp mycoides large colonies are in accordance with values reported for this organism. ${ }^{44-46}$ Although there are no clear guidelines to define effective MICs for Mycoplasma spp, interpretative criteria derived from those validated for other pathogens have been proposed. ${ }^{43}$ For example, it was speculated that the critical breakpoints for determining florfenicol efficacy against respiratory pathogens of cattle (ie, Pasteurella multocida, Mannheimia haemolytica, and Haemophilus somnus) could also represent interpretative data of Mycoplasma bovis susceptibility to florfenicol; thus, the argument could be made that when susceptibility data for Mycoplasma spp are close to those of other pathogens, they are considered predictive of potential usefulness for treatment. ${ }^{43,48}$ As such, an MIC $\leq 2 \mu \mathrm{g} / \mathrm{mL}$ would be indicative of susceptibility of Mycoplasma organisms to that antimicrobial, a value between 2 and $4 \mu \mathrm{g} / \mathrm{mL}$ would be indicative of intermediate susceptibility, and a value $\geq 8 \mu \mathrm{g} / \mathrm{mL}$ would be indicative of resistance to that antimicrobial. ${ }^{43,44}$ According to this breakpoint value, most strains of $M$ conjunctivae and $M$ agalactiae in the present study could be considered susceptible to florfenicol, and 2 strains of $M$ agalactiae could be considered resistant; however, the M mycoides subsp mycoides isolates had intermediate susceptibility.

A rational use of florfenicol to treat infectious keratoconjunctivitis in sheep should be based on sound pharmacokinetic data in the target biophase (ie, the preocular tear fluid) and pharmacodynamic data of the potential pathogens (ie, Mycoplasma spp). Florfenicol is a time-dependent antimicrobial, and the surrogate marker that best predicts its clinical efficacy is the time the concentration is higher than the MIC is during 50\% to $80 \%$ of the dosing interval. ${ }^{49}$ In the context of the present study, this pharmacokinetic-pharmacodynamic index would rely on both the bioavailability in tear fluid after parenteral administration and terminal half-life in the tear fluid. This implies that a florfenicol dosage regimen should be selected that maintains drug concentrations in tear fluid greater than the MICs of Mycoplasma spp for at least $50 \%$ of the dosing interval to optimize drug exposure for therapeutic efficacy. In the present study, only the IM route of administration achieved flo- rfenicol concentrations of at least $2 \mu \mathrm{g} / \mathrm{mL}$ in the tear fluid, and analysis of the tear concentration-time curve revealed that these concentrations were maintained for approximately 160 minutes. Thus, analysis of the data in this sample population of healthy sheep indicated that a dose of $20 \mathrm{mg} / \mathrm{kg}$, IM, every 24 hours would be unable to maintain effective drug concentrations in the tear fluid for at least $50 \%$ of the dosing interval (ie, 720 minutes) and hence would be inadequate for treatment of infectious keratoconjunctivitis caused by Mycoplasma strains.

In cattle, florfenicol can be used for the treatment of infectious keratoconjunctivitis caused by Moraxella bovis with a single SC administration of $40 \mathrm{mg} / \mathrm{kg}$ or 2 IM administrations of $20 \mathrm{mg} / \mathrm{kg} 48$ hours apart. ${ }^{28}$ Considering that $M$ bovis ( $\mathrm{MIC}$ required to inhibit growth of $90 \%$ of strains, $\leq 0.5 \mu \mathrm{g} / \mathrm{mL}$ ) are more suseptible to flofenicol than are Mycoplasma spp and that plasma clearance of florfenicol in cattle $(1.5 \mathrm{~mL} / \mathrm{kg} / \mathrm{min})$ is approximately half that in sheep $(2.6 \mathrm{~mL} / \mathrm{kg} / \mathrm{min}),{ }^{22,24}$ the plasma exposure to florfenicol in sheep would be onehalf that in cattle for the same total bioavailable dose. Consequently, the florfenicol dose should be doubled in sheep to achieve the same plasma florfenicol exposure as in cattle. Assuming that florfenicol disposition is linear, as suggested by pharmacokinetic data after administrations of 20 and $30 \mathrm{mg} / \mathrm{kg}$ in sheep, ${ }^{22}$ administration of a $40 \mathrm{mg} / \mathrm{kg}$ dose to sheep would allow florfenicol concentrations in tear fluid to be maintained at $>2 \mu \mathrm{g} /$ $\mathrm{mL}$ for approximately 320 minutes (ie, 160 minutes $X$ 2) after IM administration. The amount of time that the concentration is greater than the MIC should be at least $50 \%$ of the dosing interval; therefore, on the basis of the previous assumptions, florfenicol could be administered IM at $40 \mathrm{mg} / \mathrm{kg}$ every 12 hours for microbiological effectiveness against the most susceptible Mycoplas$m a$ isolates that cause infectious keratoconjunctivitis in sheep.

In the study reported here, florfenicol was administered IM and SC and readily penetrated the tear fluid in sheep, but analysis of the data suggested that a dose of $20 \mathrm{mg} / \mathrm{kg}$ was unlikely to be effective against the pathogenic Mycoplasma organisms involved in infectious keratoconjunctivitis in sheep. On the basis of tear concentration-time curve data and MICs of common mycoplasmal isolates, IM administration of florfenicol at a rate of $40 \mathrm{mg} / \mathrm{kg}$ every 12 hours would appear appropriate for the treatment of infectious keratoconjunctivitis in sheep. Additional studies with diseased animals and an efficacy study are needed to provide adequate treatment information. In addition, considering that we are recommending extralabel drug use, a substantially extended withdrawal period prior to marketing of milk or other edible products should be established via appropriate scientific information or veterinarians should take appropriate measures to ensure that appropriate timeframes for the withdrawal period are met to avoid violative residues.

a. Nuflor, $300 \mathrm{mg} / \mathrm{mL}$, MSD Santé Animale-Intervet, Beaucouzé, France.

b. Prednisolone Sigma P-6004, Saint Quentin Fallavier, France.

c. Inertsil ODS 3, $5 \mu \mathrm{m}, 150 \times 4.6 \mathrm{~mm}$, Interchim, Montluçon, France. 
d. Inertsil ODS3, $5 \mu \mathrm{m}, 10 \times 3.0 \mathrm{~mm}$, Interchim, Montluçon, France.

e. Glycerol, Sigma-Aldrich Inc, Saint Quentin Fallavier, France.

f. Florfenicol, Sigma-Aldrich Inc, Saint Quentin Fallavier, France.

g. Millipore, Saint Quentin en Yvelines, France.

h. Denley multipoint inoculator, Fischer Scientific Labosi, Elancourt, France.

\section{References}

1. Hosie BD. Keratoconjunctivitis in a hill sheep flock. Vet Rec 1988;122:40-43.

2. Egwu GO, Faull WB, Bradbury JM, et al. Ovine infectious keratoconjunctivitis: a microbiological study of clinically unaffected and affected sheep's eyes with special reference to Mycoplasma conjunctivae. Vet Rec 1989;125:253-256.

3. Jones GE. Infectious keratoconjunctivitis. In: Martin WB, Aitken ID, eds. Diseases of sheep. 2nd ed. London: Blackwell Scientific Publications, 1991;280-283.

4. Baas EJ, Trotter SL, Franklin RM, et al. Epidemic caprine keratoconjunctivitis: recovery of Mycoplasma conjunctivae and its possible role in pathogenesis. Infect Immun 1977;18:806-815.

5. Giacometti M, Janosvky M, Belloy L, et al. Infectious keratoconjunctivitis of ibex, chamois and other caprinae. Rev Sci Tech 2002;21:335-345

6. Dziezyc J, Millichamp NJ. Infectious ocular diseases. In: Smith BP, ed. Large animal internal medicine. 3rd ed. St Louis: Mosby, 2002;1164-1182.

7. Radostits OM, Gay CC, Hindchcliff KW, et al. Diseases of the skin, conjunctiva, and external ears. In: Radostits OM, Gay CC, Hinchcliff KW, et al. Veterinary medicine. A textbook of the diseases of cattle, horses, sheep, pigs and goats. 10th ed. Edinburgh: Saunders Elsevier; 2007;651-671.

8. Whittaker CJG, Gelatt KN, Wilkie DA. Food animal ophthalmology. In: Gelatt KN, ed. Veterinary ophthalmology. 3rd ed. Philadelphia: Lippincott Williams \& Wilkins, 1991;1117-1176.

9. Naglic T, Hajsig D, Frey J, et al. Epidemiologic and microbiological study of an outbreak of infectious keratoconjunctivitis in sheep. Vet Rec 2000;147:72-75.

10. Jones GE, Foggie A, Sutherland A, et al. Mycoplasmas and ovine keratoconjunctivitis. Vet Rec 1976;99:137-141.

11. Ter Laak EA, Schreuder BEC, Kimman TG, et al. Ovine keratoconjunctivitis experimentally induced by inoculation of Mycoplasma conjunctivae. Vet Q 1988;10:217-224.

12. Van Halderen A, Van Rensburg WJJ, Geyer A, et al. The identification of Mycoplasma conjunctivae as an aetiological agent of infectious keratoconjunctivitis of sheep in South Africa. Onderstepoort J Vet Res 1994;61:231-237.

13. Nicolet J, Freundt EA. Isolation of Mycoplasma conjunctivae from chamois and sheep affected with kerato-conjunctivitis. Zentralbl Veterinarmed B 1975;22:302-307.

14. Dagnall GJR. The role of Branhamella ovis, Mycoplasma conjunctivae and Chlamydia psittaci in conjunctivitis of sheep. Br Vet J 1994;150:65-71.

15. Motha MXJ, Frey J, Hansen MF, et al. Detection of Mycoplasma conjunctivae in sheep affected with conjunctivitis and infectious keratoconjunctivitis. N Z Vet J 2003;51:186-190.

16. Rodriguez JL, Poveda JB, Rodriguez F, et al. Ovine infectious keratoconjunctivitis caused by Mycoplasma agalactiae. Small Rumin Res 1996;22:93-96.

17. Nouws JFM, König CDW. Penetration of some antibiotics into the lacrimal fluid of sheep. Vet Q 1983;5:114-121.

18. König CDW. "Pink eye" or "zere oogies" or keratoconjunctivitis infectiosa ovis (KIO). Clinical efficacy of a number of antimicrobial therapies. Vet $Q$ 1983;5:122-127.

19. Neu HC, Fu KP. In vitro activity of chloramphenicol and thiamphenicol analogs. Antimicrob Agents Chemother 1980;18:311316.

20. Graham R, Palmer D, Pratt BC, et al. In vitro activity of florfenicol. Eur J Clin Microbiol Infect Dis 1988;7:691-694.

21. Ali BH, Al-Qarawi AA, Hashaad M. Comparative plasma pharmacokinetics and tolerance of florfenicol following intramuscular and intravenous administration to camels, sheep and goats. Vet Res Commun 2003;27:475-483.
22. Jianzhong S, Xiubo L, Haiyang J, et al. Bioavailability and pharmacokinetics of florfenicol in healthy sheep. J Vet Pharmacol Ther 2004;27:163-168.

23. Lane VM, Wetzlich S, Clifford A, et al. Intravenous and subcutaneous pharmacokinetics of florfenicol in sheep. J Vet Pharmacol Ther 2004;27:191-196.

24. Bretzlaff KN, Neff-Davis CA, Ott RS, et al. Florfenicol in nonlactating dairy cows: pharmacokinetics, binding to plasma proteins, and effect on phagocytosis by blood neutrophils. J Vet Pharmacol Ther 1987;10:233-240.

25. Lobell RD, Varma KJ, Johnson JC, et al. Pharmacokinetics of florfenicol following intravenous and intramuscular doses to cattle. J Vet Pharmacol Ther 1994;17:253-258.

26. Monaco F, Mutani R, Mastropaolo C, et al. Tears as the best practical indicator of the unbound fraction of an anticonvulsant. Epilepsia 1979;20:705-710.

27. Van Haeringen NJ. Secretion of drugs in tears. Curr Eye Res 1985;4:485-488

28. Dueger EL, Angelos JA, Cosgrove S, et al. Efficacy of florfenicol in the treatment of experimentally induced infectious bovine keratoconjunctivitis. Am J Vet Res 1999;60:960-964.

29. Angelos JA, Dueger EL, George LW, et al. Efficacy of florfenicol for treatment of naturally occurring IBK. J Am Vet Med Assoc 2000;216:62-64.

30. Gokce HI, Mehmet C, Genc O, et al. A comparison of the efficacy of florfenicol and oxytetracycline in the treatment of naturally occurring infectious bovine keratoconjunctivitis. Irish Vet J 2002;55:573-576.

31. Tang-Liu D, Schwob DL, Usansky JI, et al. Comparative tear concentrations over time of ofloxacin and tobramycin in human eyes. Clin Pharmacol Ther 1994;55:284-292.

32. Akkan AG, Mutlu I, Özyazgan S, et al. Comparative tear concentrations of topically applied ciprofloxacin, ofloxacin, and norfloxacin in human eyes. Int J Clin Pharmacol Ther 1997;35:214-217.

33. Small D, Hevy J, Tang-Liu D. Comparison of tear sampling techniques for pharmacokinetic analysis: ofloxacin concentrations in rabbit tears after sampling with Schirmer tear strips, capillary tubes, or surgical sponges. J Ocul Pharmacol 2000;16:439-446.

34. Friis NF. Selective isolation of slowly growing acidifying mycoplasmas from swine and cattle. Acta Vet Scand 1979;20:607-609.

35. Bébéar C, Robertson JA. Determination of minimal inhibitory concentration. In: Tully JG, Razin S, eds. Molecular and diagnostic procedures in mycoplasmology. Volume II: diagnostic procedures. New York: Academic Press, 1996;189-197.

36. Hannan PCT. Guidelines and recommendations for antimicrobial minimum inhibitory concentration (MIC) testing against veterinary Mycoplasma species. Vet Res 2000;31:373-395.

37. George LW, Reina-Guerra M, Baggot JD, et al. Distribution of kanamycin in ocular tissues of calves. $J$ Vet Pharmacol Ther 1986;9:183-191.

38. Woo FL, Johnson AP, Insler MS, et al. Gentamicin, tobramycin, amikacin, and netilmicin levels in tears following intravenous administration. Arch Ophthalmol 1985;103:216-218.

39. George LW, Smith JA, Kaswan R. Distribution of oxytetracycline into ocular tissues and tears of calves.J Vet Pharmacol Ther 1985;8:47-54.

40. De Craene BA, Deprez P, D'Haese E, et al. Pharmacokinetics of florfenicol in cerebrospinal fluid and plasma of calves. Antimicrob Agents Chemother 1997;41:1991-1995.

41. Pilo P, Fleury B, Marenda M, et al. Prevalence and distribution of the insertion element ISMagI in Mycoplasma agalactiae. Vet Microbiol 2003;92:37-48.

42. Marenda MS, Sagné E, Poumarat F, et al. Suppression subtractive hybridization as a basis to asses Mycoplasma agalactiae and Mycoplasma bovis genomic diversity and species-specific sequences. Microbiology 2005;151:475-489.

43. Rosenbuch RF, Kinyon JM, Apley M, et al. In vitro antimicrobial inhibition profiles of Mycoplasma bovis isolates recovered from various regions of the United States from 2002 to 2003. J Vet Diagn Invest 2005;17:436-441.

44. Ayling RD, Baker SE, Nicholas RAJ, et al. Comparison of in vitro activity of danofloxacin, florfenicol, oxytetracycline, spectinomycin and tilmicosin against Mycoplasma 
mycoides subspecies mycoides small colony type. Vet Rec 2000;146:243-246.

45. Kidanemariam A, Gouws J, van Vuuren M, et al. In vitro antimicrobial susceptibility of Mycoplasma mycoides mycoides large colony and Arcanobacterium pyogenes isolated from clinical cases of ulcerative balanitis and vulvitis in Dorper sheep in South Africa. J S Afr Vet Assoc 2005;76:204-208.

46. Al-Momani W, Nicholas RAJ, Janakat $S$, et al. The in vitro effect of six antimicrobials against Mycoplasma putrefaciens, Mycoplasma mycoides subsp. mycoides LC and Mycoplasma capricolum subsp. capricolum isolated from sheep and goats in Jordan. Trop Anim Health Prod 2006;38:1-7.
47. Antunes NT, Tavío MM, Assunção P, et al. In vitro susceptibilities of field isolates of Mycoplasma agalactiae. Vet J 2008;177:436438.

48. National Committee for Clinical Laboratory Standards. Performance standards for antimicrobial disk and dilution susceptibility tests for bacteria isolated from animals. 2nd ed. Approved standards. NCCLS document M31-A2. Wayne, Pa: National Committee for Clinical Laboratory Standards, 2002.

49. Toutain PL, Del Castillo JRE, Bousquet-Mélou A. The pharmacokinetic-pharmacodynamic approach to a rational dosage regimen for antibiotics. Res Vet Sci 2002;73:105-114. 